\title{
Early life adversity is associated with diminished social trust in adults
}

\author{
Mell, H.*, Safra, L.*, Demange, P., Algan, Y., \\ Baumard, N., Chevallier, C.
}

\begin{abstract}
Social trust is at the center of democratic societies but it varies considerably between individuals and societies, which deeply affects a range of prosocial behaviours. Socioeconomic status has been identified as an important predictor of such variability. Although this association has mostly been reported for measures of socioeconomic status taken in adulthood, recent studies have found unique effects of harsh conditions experienced during childhood on social trust assessed decades later. Here, we report a series of three studies that provide further support for the long-lasting association between early childhood conditions and social trust. The first study revealed that higher childhood socioeconomic status was associated with greater social trust in a diverse sample of French participants $(\mathrm{N}=915)$, even after adjusting for current socioeconomic status. The second study replicated this result using data from the European Values Study, an independent large-scale survey of 46 European countries $(\mathrm{N}=66,281)$. Finally, the last study found a similar association between socioeconomic status and willingness to invest in a trust game $(\mathrm{N}=60$ in original study, $\mathrm{N}=75$ in replication study).
\end{abstract}

\section{Introduction}

The degree to which people trust others in a society can affect a range of important political, economic and social outcomes. For example, individual differences in social trust are reflected in different attitudes towards the welfare state (Algan et al., 2016) and international affairs (Brewer and Steenbergen, 2002). Higher degrees of social trust have also been shown to be predict higher levels of civic engagement (Crystal and DeBell, 2002). In addition,

\footnotetext{
* equal contribution
} 
there is converging evidence in the economic literature that social trust is strongly associated with economic growth (Bjørnskov, 2017) and that social trust is an important determinant of overall well-being (Helliwell et al., 2016). Hence, it is important to learn more about the roots of social trust in order to better understand the dynamics of democratic societies.

Social trust reflects people's proneness to invest in social interactions in which potential benefits depend on others' behavior. In other words, social trust can be defined as the willingness of an individual to be vulnerable to others' actions (Mayer et al., 2006). Under this definition, social trust should vary according to people's estimation of the probability that others will actually perform the action that benefits them. The more someone believes others to be trustworthy, the more likely they should be to invest resources in social interactions in order to collect the long-term benefits of cooperation. Faced with untrustworthy social partners however, people should be less inclined to cooperate, because these partners are more likely to prefer the immediate benefits of defection over delivering a promised reward or paying a cost to achieve a shared goal. This effect of social trust on people's willingness to invest in others has been documented both in adults and children by manipulating the perceived trustworthiness of social partners, either using character vignettes, faces that vary in trustworthiness, or direct observation of trustworthy and untrustworthy behaviours (Michaelson and Munakata, 2016; Michaelson et al., 2013; Safra et al., 2016).

A consistent pattern emerging from social science studies is that differences in social trust map with individual variations in socioeconomic status, such that high socioeconomic status individuals are more likely to believe others to be trustworthy than lower socioeconomic status individuals (Alesina and La Ferrara, 2002; Brandt et al., 2015; Gheorghiu et al., 2009; Hamamura, 2012). Similarly, people with a higher socioeconomic status place more emphasis on trustworthiness when making social decisions (Safra et al., 2017). Importantly, exposure to deprivation during childhood has a long-lasting effect on social trust later in life (Hörl et al., 2016; Petersen and Aarøe, 2015). The most conclusive evidence of such long-lasting contributions of childhood conditions on social trust has been put forward by Hörl and colleagues in the context of a natural experiment in post World War II Germany (Hörl et al., 2016). After the war, Germany was divided into four occupation zones, where caloric rations were set separately every month by each of the occupying forces. Using data on temporal and regional variations in food availability, the authors demonstrated that these exogeneous variations in caloric rations during childhood had an impact on people's social trust in adulthood. Similarly, in a Danish sample, Petersen and Aarøe (Petersen and Aarøe, 2015) found an early-life effect of having low birth weight on adult 
levels of social trust, even after adjusting for a range of confounding factors. Thus, higher levels of deprivation appear to elicit greater distrust in others' prosocial intentions, with early life conditions potentially playing a key causal role.

In the current paper, we extend this work and explore the link between early socioeconomic status and social trust in a series of three studies. First, we test the relationship between childhood deprivation and adult social trust in a diverse sample of French participants $(\mathrm{N}=915)$. Second, we test the same association in a larger and more diverse sample spanning across 46 European countries $(\mathrm{N}=66,281)$. Lastly, in order to go beyond self-reported measures of social trust, we conduct an online experiment where trust is measured as participants' willingness to play in a series of trust games $(\mathrm{N}=60$ in the original study and $\mathrm{N}=75$ in the replication study).

\section{Study 1}

In Study 1, we assess the relationship between early life socio-economic status and social trust in adulthood with survey data gathered from a diverse crosssectional sample of French participants. As outlined in the introduction, we expect a positive association between childhood socioeconomic status and social trust, even after adjusting for participants' current socioeconomic status.

\subsection{Materials and Methods}

\subsubsection{Sample and procedure}

Our sample consisted of 915 French participants (482 females) aged 16 to 83 (mean: $48 \pm 0.54$ s.e.) recruited online in collaboration with the French polling institute IPSOS. Initially, a total of 11,000 participants were invited to answer a demographic survey. A subsample was then selected using quota sampling based on age, gender, geographical region, urban vs rural and occupation, to approximate a representative sample of the general French population. Selected participants had to fill a series of questionnaires including items measuring their childhood and current socioeconomic status, as well as their level of social trust. A total of 1008 participants eventually completed the whole series of questionnaires, from which 93 were excluded for giving inconsistent information about their gender. The final sample included 915 participants. 


\subsubsection{Variables of interest}

Childhood and current socioeconomic status Childhood socioeconomic status was assessed using the 3-item scale developed by Griskevicus and colleagues (2011). Specifically, participants had to report their degree of agreement on a 1-100 scale for three items: "My family usually had enough money for things when I was growing up", "I grew up in a relatively wealthy neighborhood", and "I felt relatively wealthy compared to the others kids in my school". A single index was then obtained by taking the mean of the three items $(\alpha=0.84)$.

The adult scale also included three items (Griskevicius et al., 2011): "I have enough money to buy things I want", "I don't need to worry too much about paying the bills", and "I don't think I'll have to worry about money too much in the future". Again, a single index was obtained by averaging individual scores across the three items $(\alpha=0.89)$. Participants' also provided self-reported household annual income (given on a 1-13 ordinal scale) and highest level of education (ranging from 1: Elementary school to 7: University degree). A composite index of current socioeconomic status was obtained by $z$-scoring and summing all adult socioeconomic variables.

Social trust Social trust was measured using participants' answers to the questions 'Generally speaking, would you say that most people can be trusted or that you can't be too careful in dealing with people?', 'Would you say that people usually only take care of themselves or that they try to be helpful most of the time?' and 'Do you think that most people would try to take advantage of you if they had the opportunity or that they would try to be fair?'. Answers to the first question were recoded as binary to reflect the proportion of participants believing most people to be trustworthy. The next two questions were graded on a $0-10$ scale, with higher scores reflecting a positive view of people's intentions. Participants also had the opportunity to reply I don't know to the previous two questions, in which case their answer was recoded as missing. Answers to the three items were standardized and summed to obtain a unique trust index, which we used as our dependent variable.

\subsubsection{Analysis}

All data cleaning, transformations and statistical analyses were carried out in $R$ 3.6.1 (https://www.rproject.org/), with Rstudio v1.2.5019. Using the $R$ package mice (Buuren and Groothuis-Oudshoorn, 2011), multiple imputation was performed prior to running the regression models to obtain a set of 
20 complete datasets. Regression models were then fitted to each complete dataset and the results were pooled according to Rubin's rules (Rubin, 1987).

In order to assess the relationship between childhood socioeconomic status and social trust, we fitted three separate regression models with the trust index as the dependent variable. In the first model, we only included participants' childhood socioeconomic status as a predictor. To determine whether the association could actually be completely explained by participants' current socioeconomic status, we then fitted a second model that added current socioeconomic status as an independent explanatory variable. Lastly, in a third model we examined the robustness of the association to the inclusion of classic demographic variables, i.e. participants' age and gender.

\section{$2.2 \quad$ Results}

Descriptive statistics for the variables included in the analyses are reported in Table 1, while Table 2 provides the regression estimates for the full model.

\begin{tabular}{lccccc}
\hline \hline Statistic & Mean & St. Err. & Min & Median & Max \\
\hline Age & 47.75 & 0.54 & 16 & 48 & 83 \\
Childhood socioeconomic status & 43.24 & 0.78 & 1 & 43 & 100 \\
Adult socioeconomic status & 49.52 & 0.80 & 1 & 50.33 & 100 \\
Educational level & 4.82 & 0.06 & 1 & 4 & 7 \\
Income & 8.10 & 0.09 & 1 & 8.6 & 13 \\
Current socioeconomic status & 0.00 & 0.07 & -6.65 & 0.004 & 4.75 \\
Trust index & 0.00 & 0.07 & -5.11 & -0.13 & 6.43 \\
\hline
\end{tabular}

Table 1: Descriptive statistics for the variables included in the full model (Study 1, N=915, 485 females). Childhood and Adult SES are indices calculated from the corresponding 3-items scales. Current SES is a composite index computed from the sum of Adult SES, Educational level and Income after standardization. Trust index corresponds to the sum of the standardized responses of participants to the three trust questions.

Consistent with our predictions, we found a positive association between socioeconomic status in early life and social trust in adulthood (standardized $\beta=0.13 \pm 0.03$ s.e., $t(890)=3.92, p<0.001)$. Furthermore, greater levels of social trust were still associated with higher childhood socioeconomic status once current socioeconomic status was adjusted for (childhood socioeconomic status: standardized $\beta=0.09 \pm 0.03$ s.e., $t(870)=2.74$, $p=0.006$; current socioeconomic status: standardized $\beta=0.14 \pm 0.03$ s.e., 


\begin{tabular}{lccc}
\hline \hline & Model 1 & Model 2 & Model 3 \\
\hline (Intercept) & 0.00 & 0.00 & -0.03 \\
& $(0.03)$ & $(0.03)$ & $(0.05)$ \\
Childhood socioeconomic status & $0.13^{* * *}$ & $0.09^{* *}$ & $0.12^{* *}$ \\
& $(0.03)$ & $(0.03)$ & $(0.03)$ \\
Current socioeconomic status & & $0.14^{* * *}$ & $0.13^{* * *}$ \\
Age & & $(0.04)$ & $(0.03)$ \\
Gender & & $0.22^{* * *}$ \\
& & & $0.03)$ \\
& & & 0.05 \\
& & & $(0.07)$ \\
\hline
\end{tabular}

${ }^{* * *} p<0.001,{ }^{* *} p<0.01,{ }^{*} p<0.05$

Table 2: Pooled regression coefficients for the linear models fitted on the 20 imputed datasets (Study 1, $\mathbf{N}=\mathbf{9 1 5}$ ). Coefficients reported for Childhood SES, Current SES and Age correspond to standardized estimates. Gender is coded as a binary variable (0 - male, 1 - female). Standard errors are reported inside parentheses.

$t(690)=4.06, p<0.001)$. Lastly, the inclusion of participant's age and gender as sociodemographic covariates did not alter the relationship between childhood socioeconomic status and social trust (standardized $\beta=0.11 \pm 0.03$ s.e., $t(866)=3.45, p<0.001$; see Table 2 ). Taking this estimate from the full model, an increase of one standard deviation in childhood socioeconomic status is associated with an $11 \%$ standard deviation increase on the trust index.

Hence, the results obtained from this cross-sectional analysis of a diverse sample of French participants are in line with previous findings suggesting a long-term association between levels of trusts and early life socioeconomic conditions.

\section{Study 2}

In this second study, we took advantage of available data from the European Values Study (EVS) in order to determine whether the results found in Study 1 extend to a more diverse sample. The EVS is an independent largescale sociological survey that was conducted on 66,281 respondents living in 46 different European countries (European Values Study Wave 4, European 
Values Study Longitudinal DataFile 1981-2008 (EVS 1981-2008), 2015).

\subsection{Materials and Methods}

\subsubsection{Study sample and selected variables}

The analysis reported here was performed on Wave 4 of the European Values Study (between years 2008 and 2010) and included 66281 respondents distributed in 46 countries (mean number of respondents per country: 1441 \pm 13.86 s.e.). The European Values Study questionnaire includes the three measures of social trust presented in the previous study, which were thus used in the same way to compute a trust index.

A single proxy for participants' socioeconomic status during childhood was obtained by adding up standardized answers to the questions "Parent $(s)$ had problems replacing broken things" and "Parent(s) had problems to make ends meet", both given on the same scale (1 - Yes, 2 - To some extent, 3 - A little, 4 - Not at all), as well as the highest level of education achieved by one of their parents (given on a scale ranging from 1 - Inadequately completed elementary education to 8 - University with degree).

On the other hand, participants' current socioeconomic status was captured by a composite index calculated by summing standardized scores for relative personal income (1 - Low, 2 - Middle, 3 - High) and educational level (given on the same scale as their parents' educational level).

\subsubsection{Analysis}

Again, the $R$ package mice was used to generate 20 complete datasets and the results for the regression models were pooled across imputed datasets using the mitml package. To account for the fact that participants from the EVS are nested within countries, we fitted mixed effects models with the nlme package with a random intercept and a random slope for the effect of childhood SES. As in study 1, we fitted a series of three separate models that respectively included as predictors, childhood socioeconomic status, childhood and current socioeconomic status, the two socioeconomic status indices as well as age and gender as demographic variables.

\section{$3.2 \quad$ Results}

Descriptive statistics for the variables included in the analyses are reported in Table 3, while Table 4 provides the regression estimates for the full model.

In line with the results obtained in Study 1, we found a positive association between childhood socioeconomic status and social trust in adulthood 


\begin{tabular}{lccccc}
\hline \hline Statistic & Mean & St. Err. & Min & Median & Max \\
\hline Age & 46.81 & 0.07 & 15 & 46 & 108 \\
Gender & 0.56 & 0.00 & 0 & 1 & 1 \\
$\begin{array}{l}\text { Troubles replacing } \\
\text { broken things }\end{array}$ & & & & & \\
Troubles making & 2.68 & 0.00 & 1 & 3 & 4 \\
ends meet & & & & & \\
Parents' educational & 2.75 & 0.00 & 1 & 3 & 4 \\
level & & & & & \\
Income & 3.74 & 0.01 & 1 & 3 & 8 \\
Educational level & 1.94 & 0.00 & 1 & 2 & 3 \\
Childhood socioeconomic status & 4.94 & 0.01 & 1 & 5 & 8 \\
Current socioeconomic status & 0.00 & 0.01 & -4.26 & 0.13 & 4.26 \\
Trust index & 0.00 & 0.01 & -3.20 & 0.10 & 2.89 \\
& 0.00 & 0.01 & -3.82 & -0.26 & 5.54 \\
\hline
\end{tabular}

Table 3: Descriptive statistics for the variables included in the full model (Study 2, $\mathbf{N}=\mathbf{6 6 , 2 8 1}, \mathbf{3 7 , 1 1 8}$ females) Childhood socioeconomic status is a composite index corresponding to the sum of the variables Troubles replacing broken things, Troubles making ends meet and Parents' educational level after standardization. Similarly, Current SES is a composite index computed from the variables Educational level and Income. Trust index again corresponds to the sum of the standardized responses of participants to the three trust questions. 


\begin{tabular}{lccc}
\hline \hline & Model 1 & Model 2 & Model 3 \\
\hline (Intercept) & 0.02 & 0.02 & -0.07 \\
& $(0.06)$ & $(0.06)$ & $(0.06)$ \\
Childhood socioeconomic status & $0.06^{* * *}$ & $0.03^{* * *}$ & $0.44^{* * *}$ \\
& $(0.01)$ & $(0.01)$ & $(0.01)$ \\
Current socioeconomic status & & $0.09^{* * *}$ & $0.11^{* * *}$ \\
& & $(0.01)$ & $(0.01)$ \\
Age & & & $0.07^{* * *}$ \\
Gender & & & $(0.01)$ \\
& & & $0.06^{* * *}$ \\
Random intercept & 0.18 & 0.18 & 0.17 \\
Random slope (childhood SES) & 0.002 & 0.002 & 0.002 \\
Residuals & 0.83 & 0.82 & 0.81 \\
Number of observations & & 66281 & \\
Number of groups & & 46 & \\
\hline
\end{tabular}

${ }^{* * *} p<0.001,{ }^{* *} p<0.01,{ }^{*} p<0.05$

Table 4: Pooled regression coefficients for the linear models fitted on the 20 imputed datasets (Study 2, $\mathrm{N}=66,281$ ). Coefficients reported for Childhood SES, Current SES and Age correspond to standardized estimates. Gender is coded as a binary variable (0 - male, 1 - female). Standard errors are reported inside parentheses. Variance components for the random intercept and slope for the effect of Childhood SES for the 46 countries are also reported. 
(standardized $\beta=0.06 \pm 0.007$ s.e., $t(25080)=8.42, p<0.001$ ). This association between childhood socioeconomic status and adult social trust holds when including adult socioeconomic status: while participants with higher current socioeconomic status exhibit greater social trust (standardized $\beta=0.09 \pm 0.004$ s.e., $t(870)=21.70, p<0.001$ ), a higher childhood socioeconomic status is associated with greater social trust even when adult socioeconomic status is taken into account (standardized $\beta=0.026 \pm 0.007$ s.e., $t(10952)=3.60, p<0.001)$. In the full model including participants' age and gender as covariates, the relationship is similar, with an increase of one standard deviation in childhood socioeconomic status being associated with a $4.4 \%$ standard deviation increase on the trust index (standardized $\beta=0.044 \pm 0.007$ s.e., $t(10396)=5.93, p<0.001$; see Table 4 ).

\section{$4 \quad$ Study 3}

In this section, we report results from an online study that further investigated the links between childhood/current socioeconomic status and social trust in an experimental setting. We tested whether participants with higher socioeconomic status in childhood would be more willing to invest in a series of trust games. In addition, we manipulated both the stakes of the game and reciprocation probability.

We hypothesized that individuals with a lower socioeconomic status would display decreased willingness to play, particularly in high stakes and more uncertain games, due to a lesser ability to absorb losses. This led us to the following predictions regarding our participants' behaviour in the games: i) all participants will prefer to invest in partners with a good cooperation history, yielding a positive main effect of reciprocation probability; ii) participants with a higher childhood socioeconomic status will be more willing to play the games, yielding a positive main effect of childhood SES; iii) participants with a higher childhood socioeconomic status will be more willing to invest in high stakes and more uncertain games, resulting in significant childhoodSES * stakes and childhoodSES * reciprocationprobability interactions .

The experiment was run on a sample of 60 participants, and was then replicated on an independent sample of 75 participants. 


\subsection{Materials and Methods}

\subsubsection{Overall design and procedure}

English-speaking participants were recruited via the online platform Prolific academic. Data collection was stopped once a sample of 60 participants (27 females; participants aged 18 to 63 years old, mean $=33 \pm 1.28$ s.e.) who accurately performed the 15-minutes online experiment was reached. Our a priori inclusion criteria was that participants needed to provide correct answers during the last of two training sessions, and had a reaction time above $200 \mathrm{~ms}$ on at least $90 \%$ of their trials.

Participants were paid $£ 1.5$ for their participation and could win an additional $£ 1$ bonus depending on the outcomes of the game. The experiment was programmed on Qualtrics and consisted of three independent parts: a trust game, a socio-economic and personality questionnaire, and a face evaluation task that was included to provide pilot data for an unrelated project. The questionnaires were always presented last, while the face evaluation task and trust game were presented in a random order across participants. To assess the robustness of the patterns obtained in this experiment, a replication study was conducted on a sample of 75 participants recruited on the same online platform (27 females; participants aged 18 to 65 years old, mean $=34 \pm 12$ $s d)$.

\subsubsection{Variables of interest}

Childhood and current SES Participants' childhood and current socioeconomic status were measured using the 3-item scales (Griskevicius et al., 2011) described in the first study (although answered this time on a 1-10 scale). Again, in both cases, the average across the three items was computed to obtain single indices.

Objective social trust Participants' willingness-to-play in a series of trust games was used as an objective proxy for social trust. 16 independent games were presented sequentially to the participants, involving a different partner each time. At the beginning of the experiment, participants were informed that the tokens collected at the end of the task would be transformed into a real money bonus $(1$ token $=£ 0.1)$. Then, for every game, participants received five tokens, corresponding to a bonus payment of 50 cents. They also saw a screen indicating the probability of reciprocation of the partner $(65,70$, 75 or $80 \%$ ) and the amount of tokens (1,2,3 or 5) required to play the game (i.e. what they had to give to interact with this particular social partner). For each of the sixteen games, participants had to indicate their willingness 
to play with the presented partner by answering the question "How much do you want to play with this partner?", using a 9-point scale ranging from "not at all" to "extremely". Playing the game meant accepting to transfer the associated number of tokens (i.e. the stakes) to the other player. The partner would then receive three times the amount of tokens invested, before deciding whether or not to reciprocate by giving back half of the new total of tokens to the other player, or to defect by keeping all tokens.

Importantly, in order to control for the probability of reciprocation and the stakes associated with each partner, the partners were simulated by a computer program. In order to avoid learning effects, participants did not get feedback about the partner's choice to reciprocate or not. Instead, one game was randomly selected to calculate the bonus that would be added to the participants' payment at the very end of the experiment (once participants had indicated their willingness-to-play for all 16 trust games). To do so, a random number between 1 and 9 was drawn; if this number was below the participant's willingness-to-play, the game was played and the partner's decision to reciprocate was simulated based on her reciprocation probability. If the random number was above the participant's willingness-to-play, the game was not played and the participant kept his/her 5 tokens. Finally, the resulting number of bonus tokens and the corresponding amount in pounds that would be added to the participant's payment was revealed on the final screen.

Self-reported social trust In the replication study, social trust was also measured subjectively based on participants' answer to the survey item 'Generally speaking, would you say that most people can be trusted or that you can't be too careful in dealing with people?', on a 1-10 scale.

\subsubsection{Analysis}

Individual scores for "willingness-to-play" for the sixteen games were used as our dependent measure of social trust, while stakes and probability of reciprocation of the games, childhood/current socioeconomic status and age were all standardized and used as predictors. Because willingness-to-play was measured sixteen times for each participants, individual scores were analyzed using a mixed effects linear regression model with a random intercept and random slopes on the effects of stakes and probability, with participants' ID as the grouping variable. Childhood socioeconomic status, current socioeconomic status, age and gender on the other hand were incorporated as between-subject regressors. 
To test the hypothesis of a greater sensitivity to the stakes and uncertainty among low socioeconomic status participants, interaction terms between childhood/current socioeconomic status and the parameters of the game were also included in a second model. The model without interaction was fitted to the data from both the original and the replication studies. The results for the original study are detailed in the next section. Following the approach detailed in (Braver et al., 2014), the parameters and their standards errors obtained from the replication study were used in combination with those from the original study to conduct a cumulative meta-analysis.

Finally, in addition to the previous analysis, the self-reported measure of trust available in the replication study was also analyzed using a simple linear regression model, taking childhood, current socioeconomic status, age and gender as regressors. Its correlation with average willingness-to-play is also reported.

\subsection{Results}

Descriptive statistics for the variables used in the models for the original sample are provided in Table 5 and in Table 6 for the replication sample.

\begin{tabular}{lccccc}
\hline \hline Statistic & Mean & St. Err. & Min & Median & Max \\
\hline Age & 33.43 & 1.28 & 18 & 31.5 & 63 \\
Childhood socioeconomic status & 13.62 & 0.85 & 3 & 14 & 25 \\
Adult socioeconomic status & 13.77 & 0.88 & 3 & 14 & 27 \\
$\begin{array}{l}\text { Average willingness } \\
\text { to play }\end{array}$ & 6.02 & 0.19 & 1.00 & 6.28 & 9.00 \\
\hline
\end{tabular}

Table 5: Descriptive statistics for the variables included in the original trust game experiment (Study 3, N=60, 27 females) As in the first study, Childhood and Adult SES are computed from the corresponding 3-items scale, ranging from 1 to 10 . Average willingness to play is taken across the 16 trust games series for each participant.

As expected, we found a positive main effect of reciprocation probability on willingness-to-play, meaning that participants preferred to invest in games involving partners with a good cooperation record (original sample: $\beta=$ $0.94 \pm 0.08, t(898)=11.9, p<0.001$; replication sample: $\beta=0.74 \pm 0.08$, $t(1123)=8.79, p<0.001)$. Furthermore, in line with our main hypothesis, results found in the original sample indicate that participants with a higher childhood socioeconomic status were more willing to play overall, even after 
adjusting for current socioeconomic status $(\beta=0.67 \pm 0.18, t(55)=3.78, p<$ .001). Although the association was in the same direction for the replication sample, the relationship was not significant $(\beta=0.31 \pm 0.16, t(70)=1.92, p=$ $.06)$. On the other hand, the association between childhood socioeconomic status and the declarative measure of social trust found in the replication study is consistent with our previous results $(\beta=1.01 \pm 0.30, t(70)=3.36$, $p=0.001$ ). It is worth noting that both measures of trust (behavioral and declarative) were positively correlated $(\rho=0.32, p=.005)$.

Contrary to our last prediction, there was no significant interaction of childhood socioeconomic status with either stakes or reciprocation probability: childhood SES*stakes (original sample: $\beta=-0.04 \pm 0.10$ s.e., $t(894)=-0.42, p=.67$; replication sample: $\beta=0.03 \pm 0.10, t(1119)=$ $0.29, p=.77$ ) and childhood SES*reciprocation probability (original sample: $\beta=-0.06 \pm 0.08$ s.e., $t(894)=-0.79, p=.43$; replication sample: $\beta=0.02 \pm 0.09, t(1119)=0.19, p=.85)$

Interestingly, in contrast with the first two studies, current socioeconomic status had no significant associations with the experimental measure of trust, neither as a main effect $(\beta=-0.01 \pm 0.18, t(55)=-0.03, p=.98$; replication sample: $\beta=-0.15 \pm 0.16, t(70)=-0.97, p=.34)$, nor through interaction terms: current SES*stakes (original sample: $\beta=0.16 \pm 0.10, t(894)=1.66$, $p=.10$; replication sample: $\beta=-0.03 \pm 0.10, t(1119)=-0.29, p=.77)$ and current SES*reciprocation probability (original sample: $\beta=-0.08 \pm 0.08$, $t(894)=-1.0, p=.31$; replication sample: $\beta=-0.02 \pm 0.09, t(1119)=$ $-0.2, p=.79)$.

\begin{tabular}{lccccc}
\hline \hline Statistic & Mean & St. Err. & Min & Median & Max \\
\hline Age & 34.27 & 1.46 & 18 & 33 & 65 \\
Childhood socioeconomic status & 13.72 & 0.69 & 3 & 15 & 27 \\
Adult socioeconomic status & 15.03 & 0.73 & 3 & 15 & 27 \\
Willingness to play & 6.03 & 0.15 & 2.56 & 6.25 & 9.00 \\
Self-reported social trust & 4.96 & 0.30 & 1 & 4 & 10 \\
\hline
\end{tabular}

Table 6: Descriptive statistics for the variables included in the replication trust game experiment (Study 3, N=75, 27 females) As in the first study, Childhood and Adult SES are computed from the corresponding 3 -item scale, ranging this time from 1 to 10. Average willingness to play is taken across all sixteen trust games for each participant. Self-reported social trust corresponds to participants' response to the general trust question.

Finally, the results of the cumulative meta-analysis based on the com- 
bination of the parameters and standard errors obtained from the original and replication samples, support a significant association between childhood socioeconomic status and willingness-to-play $(\beta=0.49 \pm 0.12, \mathrm{z}=4.20$, $p<0.001$; see Figure 1). All the other relationships obtained from the meta-analysis were consistent with the patterns described for the original and replication samples (Table 7 ).

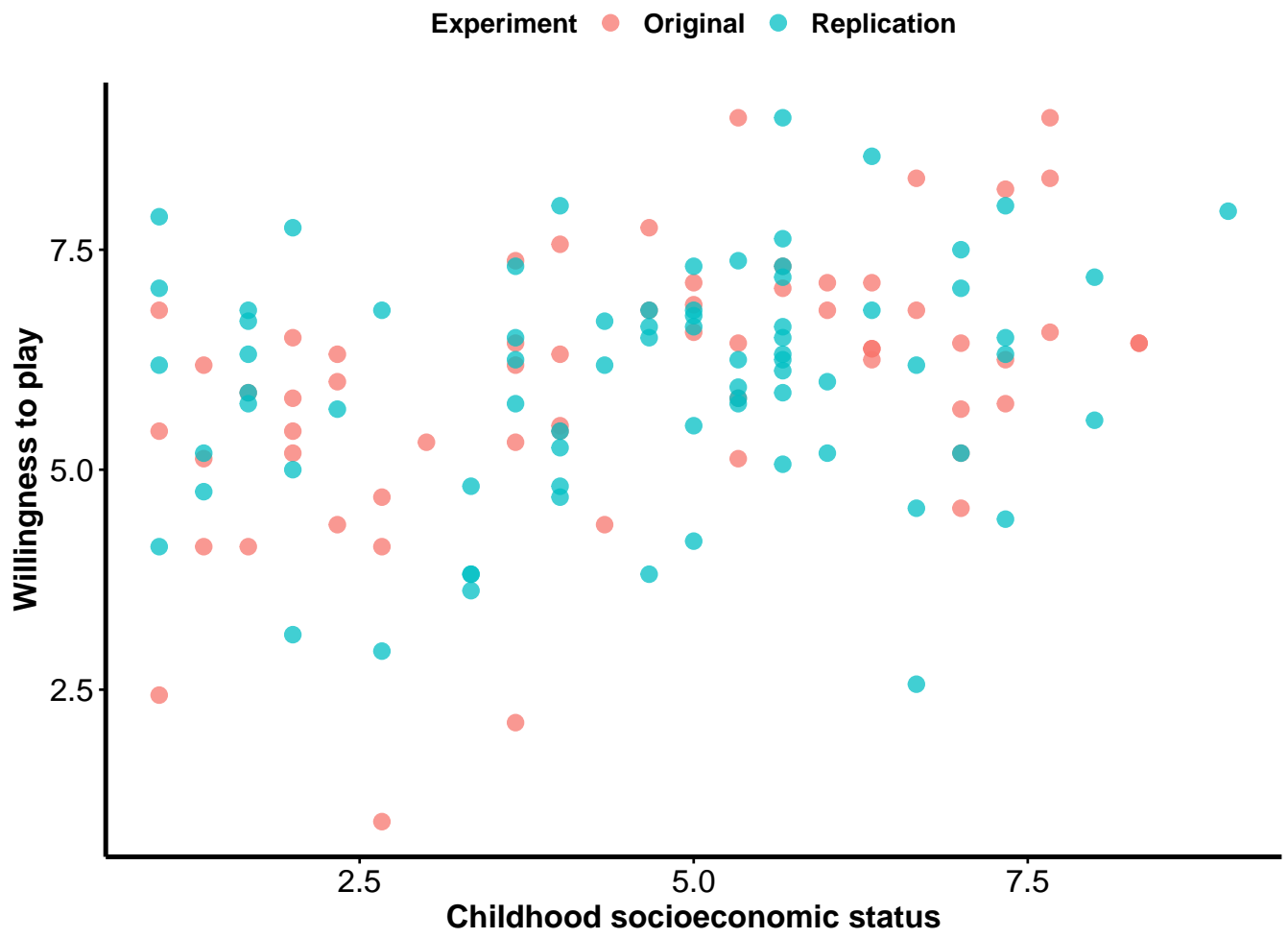

Figure 1: Participants' average willingness-to-play against childhood SES. Willingness-to-play for each participant corresponds to the average value obtained across the sixteen independent trust games, ranging from 1 to 9. Observations and statistics represented in blue and yellow are respectively for the original and the replication samples. The $R$ coefficients reported are pearson's correlation coefficients with associated $p$-values. Regression lines correspond to a simple regression model with average willingness-to-play as the dependent variable and Childhood SES index (based on the 3-items 1-10 scales) as the only regressor. 


\begin{tabular}{lcc}
\hline \hline Meta-analysis & Model 1 & Model 2 \\
\hline (Intercept) & $6.20^{* * *}$ & $6.20^{* * *}$ \\
& $(0.35)$ & $(0.35)$ \\
Childhood socioeconomic status & $0.47^{* * *}$ & $0.48^{* * *}$ \\
& $(0.12)$ & $(0.12)$ \\
Adult socioeconomic status & -0.09 & -0.07 \\
& $(0.12)$ & $(0.12)$ \\
Stake & 0.01 & -0.00 \\
Reciprocation probability & $(0.07)$ & $(0.07)$ \\
& $0.84^{* * *}$ & $0.85^{* * *}$ \\
Age & $(0.06)$ & $(0.06)$ \\
& -0.06 & -0.05 \\
Gender & $(0.11)$ & $(0.11)$ \\
Childhood SES*Stake & -0.12 & -0.12 \\
& $(0.23)$ & $(0.23)$ \\
Childhood SES*Reciprocation probability & & -0.01 \\
& & $(0.07)$ \\
Adult SES*Stake & & -0.03 \\
Adult socioeconomic status * Reciprocation probability & & $(0.06)$ \\
& & 0.07 \\
& & $(0.07)$ \\
\end{tabular}

${ }^{* * *} p<0.001,{ }^{* *} p<0.01,{ }^{*} p<0.05$

Table 7: Meta-analytic regression coefficients for the linear models fitted on the trust game data (Study 3, Original sample: $\mathrm{N}=60$, Replication sample: $\mathbf{N}=\mathbf{7 5}$ ). Model 1 includes all main effects, while model 2 adds the interactions between the parameters of the games and the socioeconomic status variables. Fixed effects estimates reported are standardized except for Gender, which is coded as a binary variable (0 - male, 1 - female). Standard errors are reported inside parentheses.

\section{General discussion}

The studies reported in this paper investigate the relationship between social trust and early life socioeconomic status. In all three studies, we found a consistent association between lower socioeconomic status during childhood and lower social trust during adulthood, even after adjusting for participants' current socioeconomic status, as well as age and gender. Besides self-reported measures of social trust, we also found an association between participants' 
childhood socioeconomic status and their willingness to invest in a trust game. This finding suggests that the relationship observed between childhood socioeconomic status and social trust measured via subjective questionnaires can be extended to actual behaviours in economic games.

These results are in line with earlier findings regarding the origins of social trust (Hörl et al., 2016; Petersen and Aarøe, 2015) and further support the emerging consensus that higher levels of cooperation are associated with higher socioeconomic status in a range of settings (dictator game, prisoner's dilemma, lost letter, declarative reports and questionnaires; see for example Brandt et al. (2015), Falk and Zehnder (2007), Holland et al. (2012), Korndörfer et al. (2015), Nettle et al. (2011), Safra et al. (2017), and Silva and Mace $(2014,2015)$ ). Overall, these empirical results are of central importance to understand the dynamics of democratic societies because they provide suggestive evidence of a long-lasting association between socioeconomic conditions and social and political behavior.

Several psychological mechanisms might underlie this association. One compelling hypothesis is that exposure to deprivation biases individuals' preferences towards short-term benefits (Pepper and Nettle, 2017). Empirically, this idea is backed by multiple studies showing a correlation between higher socioeconomic status and decreased delay discounting. For instance, Green et al. (1996), Harrison et al. (2002), and Bruderer Enzler et al. (2014) found less discounting among high-income participants. A similar trend has also been documented with individuals' level of education, such that a higher level of education is associated with lower temporal discounting (Bruderer Enzler et al., 2014; Jaroni et al., 2004; Lee et al., 2013).

Theoretically, this shift towards more immediate rewards in deprived ecologies can reflect an appropriate response to an unpredictable future (Pepper and Nettle, 2017) or high waiting costs due to a lack of capital (Mell et al., $2017,2019)$. In both cases, the association between deprivation and shorter time horizon is likely to affect social trust. Mathematical models of human cooperation by reciprocity suggest that variations in cooperation might depend on individuals' time preferences (Leimar and Hammerstein, 2001; Nowak and Sigmund, 2005). The reason is that cooperation is a futureoriented strategy: in the short-term, it is more advantageous to cheat others and get an immediate but smaller benefits. In the longer term, however, it is more advantageous to refrain from cheating, have a good reputation, and reap the more distant but greater benefits of sustained cooperation. In line with this idea, a number of studies have demonstrated an association between patience and cooperation in the lab as well as in large scale surveys on real-life attitudes and behaviours (Curry et al., 2008; Harris and Madden, 2002; Lettinga et al., 2019). 
Another causal channel between deprivation and trust is risk aversion. Empirical studies demonstrate that risk aversion for a range of investments decreases with socioeconomic status (Amir et al., 2018; Haushofer and Fehr, 2014), which may reflect the fact that a greater access to resources provides a buffer against losses, thus reducing individuals' sensitivity towards risky investments. In the social realm, a lower level of risk aversion might lead to a higher level of social trust by reducing the perceived cost of misjudging social partners and being cheated in cooperative interactions. On the other hand, because lower socioeconomic status individuals are less able to absorb losses when partners do not cooperate, they should also be more risk-averse in social situations. This perspective predicts that lower socioeconomic status people will err on the side of caution and be more biased to believe that others are generally untrustworthy. The true proportion of selfish individuals might not correspond to their belief, but this error management strategy (Haselton and Nettle, 2006) would contribute to shielding them from the costs of exploitation, which is proportionally greater for them than it is for people with more resources. According to this view, people living in lower socioeconomic status neighborhoods should adjust their social trust levels to match an environment where cooperation is truly more risky.

Regarding the causal role of risk, we hypothesized that individuals of low socioeconomic status would be less willing to engage in high-risk (i.e. low-reciprocation probability) and high-stakes interactions due to a lesser ability to absorb losses. Yet, we did not find supporting evidence for an interaction between socioeconomic status and participants' sensitivity to the risk and stakes of the games. Additional research is therefore needed to test whether other designs might be better suited to explore these more finegrained properties of risk management strategies.

Overall, there are theoretical and empirical reasons to predict that differences in both time and risk preferences among people of varying socioeconomic status should have convergent effects on social trust. Our results are in line with this broad framework and suggest that social trust could be added to the growing list of social, economic and individual behaviours that are impacted by early life adversity (Pepper and Nettle, 2017). Importantly, our results also provide suggestive evidence that this effect is not circumscribed in time but that there are potentially long-lasting effects of economic changes on political and social attitudes. Future research should investigate whether these results generalize beyond generalized social trust and extend to individuals various social circles (e.g., family, foreigners or the government). Prior research has indeed shown that the level of trust in different social circles are associated with different political outputs (Hetherington and Husser, 2012; Wals et al., 2015). 
At the population level, ours results could help explain why higher economic development is associated with a higher level of social trust (Bjørnskov, 2017; Ortiz-Ospina and Roser, 2020) and with the development of democratic regimes (Barro, 1999; Epstein et al., 2006; Papaioannou and Siourounis, 2008). As living standards and life expectancy increase, individuals become less suspicious and more open to cooperating with others, hence increasing their level of social trust. Ultimately, increased social trust may lead to broader changes at the population level, and could facilitate the rise of modern democratic regimes across the world (Inglehart, 1997) that are based on high level of social trust (Baumard, 2019; Safra et al., 2020).

\section{References}

Alesina, A. and E. La Ferrara (2002). "Who Trusts Others?" In: Journal of public economics 85.2, pp. 207-234.

Algan, Y., P. Cahuc, and M. Sangnier (2016). "Trust and the Welfare State: The Twin Peaks Curve". In: The Economic Journal 126.593, pp. 861883. ISSN: 1468-0297. DOI: 10.1111/ecoj.12278.

Amir, D., M. R. Jordan, and D. G. Rand (2018). "An Uncertainty Management Perspective on Long-Run Impacts of Adversity: The Influence of Childhood Socioeconomic Status on Risk, Time, and Social Preferences". In: Journal of Experimental Social Psychology 79, pp. 217-226. ISSN: 00221031. DOI: 10.1016/j . jesp. 2018.07.014.

Barro, R. J. (1999). "Determinants of democracy". In: Journal of Political economy 107.S6, S158-S183.

Baumard, N. (2019). "Psychological origins of the Industrial Revolution". In: Behavioral and Brain Sciences 42, e189. DOI: 10.1017/S0140525X1800211X.

Bjørnskov, C. (2017). Social Trust and Economic Growth. SSRN Scholarly Paper ID 2906280. Rochester, NY: Social Science Research Network.

Brandt, M. J., G. Wetherell, and P. J. Henry (2015). "Changes in Income Predict Change in Social Trust: A Longitudinal Analysis". In: Political Psychology 36.6, pp. 761-768. ISSN: 1467-9221. DOI: $10.1111 /$ pops . 12228.

Braver, S. L., F. J. Thoemmes, and R. Rosenthal (2014). "Continuously cumulating meta-analysis and replicability". In: Perspectives on Psychological Science 9.3, pp. 333-342.

Brewer, P. R. and M. R. Steenbergen (2002). "All against all: How beliefs about human nature shape foreign policy opinions". In: Political Psychology 23.1, pp. 39-58. 
Bruderer Enzler, H., A. Diekmann, and R. Meyer (2014). "Subjective Discount Rates in the General Population and Their Predictive Power for Energy Saving Behavior". In: Energy Policy 65, pp. 524-540. ISSN: 03014215. DOI: $10.1016 / \mathrm{j}$. enpol.2013.10.049.

Buuren, S. van and K. Groothuis-Oudshoorn (2011). "Mice: Multivariate Imputation by Chained Equations in $R$ ". In: Journal of Statistical Software 45.3. ISSN: 1548-7660. DOI: 10.18637/jss.v045.i03.

Crystal, D. S. and M. DeBell (2002). "Sources of Civic Orientation Among American Youth: Trust, Religiious Valuation, and Attributions of Responsibility". In: Political Psychology 23.1, pp. 113-132.

Curry, O. S., M. E. Price, and J. G. Price (2008). "Patience Is a Virtue: Cooperative People Have Lower Discount Rates". In: Personality and Individual Differences 44.3, pp. 780-785. ISSN: 0191-8869. DOI: 10.1016/ j.paid.2007.09.023.

Epstein, D. L. et al. (2006). "Democratic transitions". In: American journal of political science 50.3, pp. 551-569.

Falk, A. and C. Zehnder (2007). Discrimination and In-Group Favoritism in a Citywide Trust Experiment. 2765. Institute of Labor Economics (IZA).

Gheorghiu, M. A., V. L. Vignoles, and P. B. Smith (2009). "Beyond the United States and Japan: Testing Yamagishi's Emancipation Theory of Trust across 31 Nations". In: Social Psychology Quarterly 72.4, pp. 365383. ISSN: 0190-2725, 1939-8999. DOI: 10.1177/019027250907200408.

Green, L. et al. (1996). "Temporal Discounting in Choice between Delayed Rewards: The Role of Age and Income". In: Psychology and Aging 11.1, pp. 79-84. ISSN: 0882-7974. DOI: 10.1037//0882-7974.11.1.79.

Griskevicius, V. et al. (2011). "Environmental Contingency in Life History Strategies: The Influence of Mortality and Socioeconomic Status on Reproductive Timing." In: Journal of Personality and Social Psychology 100.2, pp. 241-254. ISSN: 1939-1315, 0022-3514. DOI: 10.1037/a0021082.

Hamamura, T. (2012). "Social Class Predicts Generalized Trust But Only in Wealthy Societies". In: Journal of Cross-Cultural Psychology 43.3, pp. 498-509. ISSN: 0022-0221, 1552-5422. DOI: 10.1177/0022022111399649.

Harris, A. C. and G. J. Madden (2002). "Delay Discounting and Performance on the Prisoner's Dilemma Game". In: The Psychological Record 52.4, pp. 429-440. ISSN: 2163-3452. DOI: 10.1007/BF03395196.

Harrison, G., M. Lau, and M. B. Williams (2002). "Estimating Individual Discount Rates in Denmark: A Field Experiment". In: American Economic Review 92.5, pp. 1606-1617.

Haselton, M. G. and D. Nettle (2006). "The Paranoid Optimist: An Integrative Evolutionary Model of Cognitive Biases". In: Personality and So- 
cial Psychology Review 10.1, pp. 47-66. ISSN: 1088-8683, 1532-7957. DOI: 10.1207/s15327957pspr1001_3.

Haushofer, J. and E. Fehr (2014). "On the Psychology of Poverty". In: Science 344.6186, pp. 862-867. ISSN: 0036-8075, 1095-9203. DOI: $10.1126 /$ science. 1232491.

Helliwell, J. F., H. Huang, and S. Wang (2016). New Evidence on Trust and Well-Being. Working Paper 22450. National Bureau of Economic Research. DOI: 10.3386/w22450.

Hetherington, M. J. and J. A. Husser (2012). "How trust matters: The changing political relevance of political trust". In: American Journal of Political Science 56.2, pp. 312-325.

Holland, J., A. S. Silva, and R. Mace (2012). "Lost Letter Measure of Variation in Altruistic Behaviour in 20 Neighbourhoods". In: PLoS ONE 7.8. Ed. by Y. Moreno, e43294. ISSN: 1932-6203. DOI: 10 . 1371/ journal . pone. 0043294.

Hörl, M. et al. (2016). Early-Life Circumstances Predict Measures of Trust Among Adults: Evidence from Hunger Episodes in Post-War Germany. SSRN Scholarly Paper ID 2866849. Rochester, NY: Social Science Research Network.

Inglehart, R. (1997). Modernization and Postmodernization: Cultural, Economic, and Political Change in 43 Societies. Princeton, N.J: Princeton University Press. 453 pp. ISBN: 978-0-691-01181-3 978-0-691-01180-6.

Jaroni, J. L. et al. (2004). "Relationship between Education and Delay Discounting in Smokers". In: Addictive Behaviors 29.6, pp. 1171-1175. ISSN: 0306-4603. DOI: $10.1016 / \mathrm{j}$. addbeh. 2004.03.014.

Korndörfer, M., B. Egloff, and S. C. Schmukle (2015). "A Large Scale Test of the Effect of Social Class on Prosocial Behavior". In: PLOS ONE 10.7. Ed. by M. Espinosa, e0133193. ISSN: 1932-6203. DOI: 10.1371/journal. pone. 0133193.

Lee, N. C. et al. (2013). "Age and Educational Track Influence Adolescent Discounting of Delayed Rewards". In: Frontiers in Psychology 4. ISSN: 1664-1078. DOI: $10.3389 / \mathrm{fpsyg} .2013 .00993$.

Leimar, O. and P. Hammerstein (2001). "Evolution of Cooperation through Indirect Reciprocity". In: Proceedings of the Royal Society of London. Series B: Biological Sciences 268.1468, pp. $745-753$. DOI: $10.1098 / \mathrm{rspb}$. 2000.1573.

Lettinga, N. et al. (2019). "Environmental Harshness Is Associated with Lower Investment in Collective Actions". In: bioRxiv, p. 663518. DOI: $10.1101 / 663518$. 
Mayer, R. C., J. H. Davis, and F. D. Schoorman (2006). "An integrative model of organizational trust". In: Organizational trust: A reader, pp. 82 108.

Mell, H., N. Baumard, and J.-B. André (2017). "Both Collection Risk and Waiting Costs Give Rise to the Behavioral Constellation of Deprivation". In: Behavioral and Brain Sciences 40. ISSN: 0140-525X, 1469-1825. DOI: 10.1017/S0140525X17001030.

- (2019). "Time Is Money. Waiting Costs Explain Why Selection Favors Steeper Time Discounting in Deprived Environments." In: DOI: 10.32942/ osf .io/7d56s.

Michaelson, L. E. and Y. Munakata (2016). "Trust Matters: Seeing How an Adult Treats Another Person Influences Preschoolers' Willingness to Delay Gratification". In: Developmental Science 19.6, pp. 1011-1019. ISSN: 1467-7687. DOI: 10.1111/desc. 12388.

Michaelson, L. et al. (2013). "Delaying Gratification Depends on Social Trust". In: Frontiers in Psychology 4, p. 355. ISSN: 1664-1078. DOI: $10.3389 /$ fpsyg. 2013.00355.

Nettle, D., A. Colléony, and M. Cockerill (2011). "Variation in Cooperative Behaviour within a Single City". In: PLoS ONE 6.10. Ed. by Y. Moreno, e26922. ISSN: 1932-6203. DOI: 10.1371/journal . pone.0026922.

Nowak, M. A. and K. Sigmund (2005). "Evolution of Indirect Reciprocity". In: Nature 437.7063, pp. 1291-1298. ISSN: 1476-4687. DOI: 10.1038 / nature 04131.

Ortiz-Ospina, E. and M. Roser (2020). "Trust". In: Our World in Data. https://ourworldindata.org/trust.

Papaioannou, E. and G. Siourounis (2008). "Democratisation and growth". In: The Economic Journal 118.532, pp. 1520-1551.

Pepper, G. V. and D. Nettle (2017). "The Behavioural Constellation of Deprivation: Causes and Consequences". In: Behavioral and Brain Sciences 40. ISSN: 0140-525X, 1469-1825. DOI: 10.1017/S0140525X1600234X.

Petersen, M. B. and L. Aarøe (2015). "Birth Weight and Social Trust in Adulthood: Evidence for Early Calibration of Social Cognition". In: Psychological Science 26.11, pp. 1681-1692. ISSN: 0956-7976. DOI: 10.1177/ 0956797615595622.

Rubin, D. B., ed. (1987). Multiple Imputation for Nonresponse in Surveys. Wiley Series in Probability and Statistics. Hoboken, NJ, USA: John Wiley \& Sons, Inc. ISBN: 9780470316696 . DOI: 10.1002/9780470316696.

Safra, L. et al. (2016). "Neighborhood Deprivation Negatively Impacts Children's Prosocial Behavior". In: Frontiers in Psychology 7. ISSN: 1664-1078. DOI: $10.3389 /$ fpsyg . 2016.01760. 
Safra, L. et al. (2017). "Childhood Harshness Predicts Long-Lasting Leader Preferences". In: Evolution and Human Behavior 38.5, pp. 645-651. ISSN: 10905138. DOI: $10.1016 / \mathrm{j}$. evolhumbehav . 2017.05.001.

Safra, L. et al. (2020). "(under revision) Tracking the rise of trust in history using machine learning and paintings". In:

Silva, A. S. and R. Mace (2014). "Cooperation and Conflict: Field Experiments in Northern Ireland". In: Proceedings of the Royal Society B: Biological Sciences 281.1792, p. 20141435. ISSN: 0962-8452, 1471-2954. DOI: 10.1098/rspb. 2014.1435.

- (2015). "Inter-Group Conflict and Cooperation: Field Experiments Before, During and After Sectarian Riots in Northern Ireland". In: Frontiers in Psychology 6. ISSN: 1664-1078. DOI: 10.3389/fpsyg.2015.01790.

Wals, S. C. et al. (2015). "Love thy neighbor? Trust in foreigners and support for transnational policies". In: Political Research Quarterly 68.3, pp. 537551. 